\title{
Maximum Cellular Boolean Functions and Perfect Gray Codes ${ }^{1}$
}

\author{
A. J. Goldman and B. K. Bender
}

(January 28, 1963)

\begin{abstract}
I. A Boolean function of $n$ variables, considered as a subset of the discrete unit $n$-cube $B_{n}$, is called cellular if each of its connected components is a face of $B_{n}$. Hamming's determination of optimal binary single-error-detecting codes is generalized to a characterization of all proper cellular functions with the greatest possible number of elements. II. An analysis is made of a class of Gray codes (Hamiltonian circuits on $B_{n}$ ) with certain special properties, e.g., admitting for $0 \leq d \leq n$ a partition into $2^{n-d}$ subpaths each forming a $d$-dimensional face of $B_{n}$.
\end{abstract}

\section{Introduction}

Let $B_{n}$ be the set of vertices of the unit cube in $n$-dimensional space; equivalently, $B_{n}$ is the set of all tbinary sequences ${ }^{2}$ of length $n$. A Boolean function of $n$ variables is simply a subset ${ }^{3}$ of $B_{n}$.

$*$ Call two vertices of $B_{n}$ neighbors if they differ in precisely one position. A Boolean function $f$ is called connected if, given any two vertices $X, X^{\prime}$ in $f$, there exists a sequence

$$
X=X_{1}, X_{2}, X_{3}, \ldots, X_{k-1}, X_{k}=X^{\prime}
$$

of members of $f$ such that $X_{i}$ and $X_{i+1}$ are neighbors for $1 \leq i \leq k-1$. It is easy to show that if $f$ is not connected, then it has a unique partition into maximal connected subsets called its components; ${ }^{4}$ if $f$ is connected, the unique component consists of $f$ itself.

The relevance of these concepts to the simplification of Boolean functions is as follows. By a cell of a Boolean function $f$, we mean a face ${ }^{5}$ of $B_{n}$ which lies wholly in $f$. A cover of $f$ is a collection $C$ of cells of $f$ such that each vertex of $f$ lies in at least one cell from $C$. Each cell of $f$ is assigned a cost in a manner which need not be described here, and the cost of a cover $C$ is deiined to be the sum of the costs of the cells of $C$. The problem of finding a minimum-cost cover of $f$ is then identical with the "Problem of Quine" 6 of finding a simplest "normal disjunctive form" for $f$, a problem of practical interest (for example) in the design of switching circuits. From the fact that each cell is connected

\footnotetext{
${ }^{1}$ Supported in part by the National Science Foundation, Grant No. G-7579. 2 A sequence is binary if each of its terms is either 0 or 1 .

3 The equivalence of this definition with alternative ones is well-known in the field and will not be discussed here

4 Define a binary relation on $f: X R X^{\prime}$ holds if $X$ and $X^{\prime}$ are in a connected subset of $f$. Then $R$ is an equivalence relation and the components of $f$ are the corresponding equivalence classes.

corresponding equivalence classes. (binary) values of $n-d$ of the $n$ coordinates.

(binary) values of $n-d$ of the $n$ coordinates. Monthly 59 (1952), pp. 521-531. There is no reason here to eite the extensive literature on this topic.
}

and therefore lies in a single component of $f$, it follows that any cover of $f$ is a disjoint union of covers of the components of $f$ and conversely; in particular any minimum-cost cover of $f$ is a disjoint union of minimum-cost covers of the components of $f$, and conversely. That is, the Boolean simplification problem for a function $f$ which is not connected, can be split into independent "smaller" subproblems by splitting $f$ into its components. Such a decomposition is clearly likely to be helpful in attacking the simplification problem. ${ }^{7}$

In this context it is natural to ask how many components a Boolean function of $n$ variables can possibly have. This question will be answered in section 2 , in which we also investigate the structure of the maximum disconnected functions of $n$ variables, i.e., those with the greatest possible number of components.

The discussion above also makes it natural to focus attention on classes of Boolean functions $f$ such that the simplification problem for each component of $f$ is especially easy. One such class is that of isolated functions, those with only single vertices of $B_{n}$ as components. An isolated Boolean function can be identified with a single-error-detecting binary code, and our characterization in section 2 of maximum isolated functions (those with as many vertices as possible), which verifies a conjecture of Chittenden ${ }^{8}$ is essentially the same as an "optimal code" characterization due to Hamming. ${ }^{9}$

For all methods of "cost" assignment suggested for the Problem of Quine, the faces $F$ of $B_{n}$ have the property that $\{F\}$ is the unique minimum-cost cover of $F$. Thus the class of Boolean functions $f$, such that each component of $f$ is a cell of $f$, is one of the

7 Of course the labor required to determine the components should be taken into account.

$8 \mathrm{E}$. W. Chittenden, On the minimal representation of Boolean functions, Transactions of the Third Conference of Arsenal Mathematicians, U.S. Army Office of Ordnance Research Rept. No. 58-2, p. 134.

Ore of Coll System Tech. J. 29 (1950), pp. 147-160. Our proof by induction is quite different from Hamming's, and this approach may be of independent interest. 
classes mentioned in the last paragraph. Such functions will be called cellular, and in section 3 we determine the structure of maximum cellular functions (those other than $B_{n}$, with as many vertices as possible). This generalization of Hamming's theorem from isolated functions to cellular functions, is the main new result of the paper.

A Gray code on $B_{n}$, in the language of graph theory, is a Hamiltonian circuit of the graph formed from $B_{n}$ by the "neighbor" relation. ${ }^{9 \mathrm{a}}$ In section 4 , the inductive approach introduced earlier (in sec. 2) is employed to establish the existence of Gray codes with certain special properties, e.g., admitting a partition into $2^{\mathrm{n}-\mathrm{d}}$ subpaths each forming a $d$-dimensional face of $B_{n}$. These "perfect" Gray codes turn out to coincide with what were called the "conventional" Gray codes by Gilbert, ${ }^{9 b}$ who described them in different notation using essentially the same recursive construction. The treatment in section 4 , besides giving additional detail on the structure of these Hamiltonian circuits, highlights their geometric aspects in a way which may be better suited to some readers' mathematical intuition than is the strictly arithmetic treatment associated in this context with the phrase "reflected binary." may be of interest that this material was developed from purely geometric considerations, in ignorance of "reflected binary."

\section{Maximum Disconnected and Isolated Functions}

We begin with a simple lemma:

Lemma 1. There is at least one maximum disconnected function of $\mathrm{n}$ variables which is isolated.

For the proof, observe first that because the number of Boolean functions of $n$ variables is finite, a maximum disconnected function $f$ must exist. Now form a function $f^{\prime}$ by deleting all but one member from every component of $f$. Clearly $f^{\prime}$ is isolated, and it must be maximum disconnected since it has the same number of components as does $f$.

In what follows, it will be convenient to associate to each Boolean function $f$ of $k+1$ variables, two Boolean functions $f_{0}$ and $f_{1}$ of $k$ variables each, called the projections ${ }^{9 \mathrm{~d}}$ of $f . f_{0}$ is obtained by dropping the final 0 from each member of $f$ which ends in 0 , while $f_{1}$ is obtained by dropping the final 1 from each member of $f$ which ends in 1 . Note that

$$
|f|=\left|f_{0}\right|+\left|f_{1}\right|
$$

where $|f|$ denotes the number of members of $f$.

LeMma 2. If $\mathrm{M}_{\mathrm{n}}$ is the maximum number of components of a Boolean function of $\mathrm{n}$ variables, then $\mathrm{M}_{\mathrm{n}} \leq 2^{\mathrm{n}-1}$.

9a Some authors use "Gray code" to mean any path or circuit in the linear graph formed by $B_{n}$.

${ }_{9 \mathrm{~b}}$ E. C. Gilbert, Gray codes and paths on the $N$-cube, Bell System Tech. J. 37 (1958).

Eng. Vol. EC-5 (1956)

$\theta \mathrm{d}$ In the switching-circuit literature $f_{0}$ and $f_{1}$ would be called the residues of $f$ with respect to the "last" of the $k+1$ Boolean variables.
This is clear for $n=1$; assume it true for $n=k$ and consider the case $n=k+1$. Lemma 1 guarantees the existence of a maximum disconnected function $f$ of $k+1$ variables which is isolated; then eq (1) shows that $M_{k+1}=\left|f_{0}\right|+\left|f_{1}\right|$. It is easy to see that $f_{0}$ and $f_{1}$ are also isolated, so that for $i=0$ and $i=1,\left|f_{i}\right|$ is the number of components of $f_{i}$. Therefore $\left|f_{i}\right| \leq M_{k}$, and so we have $M_{k+1} \leq 2 M_{k}$. But $M_{k} \leq 2^{k-1}$ by the induction hypothesis, so that $M_{k+1} \leq 2^{k}$ and the induction proof is complete.

We now define two special Boolean functions of $n$ variables; $f(n)$ consists of those members of $B_{n}$ with an even number (possibly zero) of 1's among their entries, ${ }^{9 \mathrm{e}}$ while $g(n)$ consists of those members of $B_{n}$ with an odd number of 1's among their entries.

TheOrem 1. The functions $\mathrm{f}(\mathrm{n})$ and $\mathrm{g}(\mathrm{n})$, which form a partition of $\mathrm{B}_{\mathrm{n}}$, are the only maximum isolated Boolean functions of $\mathrm{n}$ variables. $\mathrm{M}_{\mathrm{n}}=2^{\mathrm{n}-1}$.

Part of the proof is trivial; it is clear that $f(n)$ and $g(n)$ are isolated, and that they form a partition of $B_{n}$. Since $f(n)$ and $g(n)$ each have $2^{n-1}$ members and thus the same number of components, we have $M_{n} \geq 2^{n-1}$ and the equality follows by lemma 2 .

It only remains to show that if $h$ is any maximum isolated function of $n$ variables, then $h$ is either $f(n)$ or $g(n)$. This is clear for $n=1$; suppose it true for $n=k$ and consider the case $n=k+1$. As in the proof of lemma 2 ,

$$
2^{k}=M_{k+1}=|h|=\left|h_{0}\right|+\left|h_{1}\right| \leq M_{k}+M_{k}=2^{k},
$$

where we have used the fact that $h_{0}$ and $h_{1}$ are isolated because $h$ is. Since equality holds throughout the last display, $h_{0}$ and $h_{1}$ each have exactly $M_{k}$ components. The induction hypothesis leads to four possibilities:

$$
\begin{aligned}
& h_{0}=f(k) \text { and } h_{1}=f(k), \\
& h_{0}=g(k) \text { and } h_{1}=g(k), \\
& h_{0}=f(k) \text { and } h_{1}=g(k), \\
& h_{0}=g(k) \text { and } h_{1}=f(k) .
\end{aligned}
$$

The first two possibilities are incompatible with the fact that $h$ is isolated, the third yields $h=f(k+1)$, and the fourth yields $h=g(k+1)$; these conclusions follow from the identity ${ }^{10}$

$$
h=\left(h_{0} \times\{0\}\right) \cup\left(h_{1} \times\{1\}\right)
$$

valid for Boolean functions of $k+1$ variables. Thus the induction proof is complete.

[Jack Edmonds (NBS Operations Research Section) has observed that $B_{n}$ can be regarded as a bipartite graph, and has given the following (unpublished) generalization of theorem 1: For a connected bipartite graph which has a connected regular subgraph containing all its vertices, the sets of the

\footnotetext{
๑e That is, a vertex $X=\left(x_{1}, x_{2}, \ldots, x_{n}\right)$ of $B_{n}$ is in $f(n)$ if and only if $\sum_{i=1}^{n} x_{i}$ is even.

${ }_{10} h_{0} \times\{0\}$ is obtained by adjoining a 0 at the end of each member of $h_{0}$. The multiplication sign refers to the Cartesian-product representation of $B_{k+1}$ as ${ }_{B} \times\{0,1\}$
} 
unique bipartition are the only maximum isolated sets of vertices.]

One might conjecture that a maximum disconnected Boolean function is necessarily isolated. A counter-example for $n=1$ is provided by $B_{1}$ itself. The next theorem shows that this is the only counterexample.

TheOrem 2. For $\mathrm{n}>1$, every maximum disconnected function of $\mathrm{n}$ variables is isolated.

To prove this, let $f$ be a maximum disconnected function of $n>1$ variables. As in the proof of lemma 1 , form $f^{\prime}$ by deleting all but one member from each component of $f$. Since $f^{\prime}$ is isolated and has the same number of components as $f$, it follows from theorem 1 that $f^{\prime}$ is either $f(n)$ or $g(n)$. Without loss of generality assume $f^{\prime}=f(n)$. If $f \neq f^{\prime}$, then $f$ includes at least one vertex $Y$ of $g(n)$.

One neighbor $X_{1}$ of $Y$ is obtained by changing just the first entry of $Y$, while a second neighbor $X_{2}$ is obtained by changing just the second entry of $Y$. Since $X_{1}$ and $X_{2}$ are in $f(n)=f^{\prime}$ and thus in $f$, and both are neighbors of the same vertex $Y$ of $f$, it follows that the elements $X_{1}$ and $X_{2}$ of $f^{\prime}$ lie in the same component of $f$. This however contradicts the construction of $f^{\prime}$; we conclude that $f=f^{\prime}$, so that $f$ is isolated.

\section{Maximum Cellular Functions}

Recall that a Boolean function $f$ is called cellular if each component of $f$ is a cell of $f$; different components may be cells of different dimensions. Cellular functions are a natural generalization of jsolated functions.

Theorem 3. Let $\mathrm{F}_{\mathrm{d}}$ be a d-dimensional face of $\mathrm{B}_{\mathrm{n}}, 0 \leq \mathrm{d}<\mathrm{n}$. There exists a Boolean function $\mathrm{f}\left(\mathrm{n} ; \mathrm{F}_{\mathrm{d}}\right)$ of $\mathrm{n}$ variables with $\mathrm{F}_{\mathrm{d}}$ as a component, $2^{\mathrm{n}-\mathrm{d}-1}$ components in all, and every component a d-dimensional cell. The complement $\mathrm{g}\left(\mathrm{n} ; \mathrm{F}_{\mathrm{d}}\right)$ of $\mathrm{f}\left(\mathrm{n} ; \mathrm{F}_{\mathrm{d}}\right)$ in $\mathrm{B}_{\mathrm{n}}$ also has $2^{\mathrm{n}-\mathrm{d}-1}$ components, each a d-dimensional cell.

These functions $f\left(n ; F_{d}\right)$ and $g\left(n ; F_{\mathrm{d}}\right)$ can be regarded as the analogs, for cellular functions, of the functions $f(n)$ and $g(n)$ (defined before theorem 1) for isolated functions. For example our later results will imply as a special case that these functions (for various $F_{d}$ ) give the only solutions to the problem of constructing a cellular function with as many components as possible under the restriction that every component is a $d$-dimensional cell; theorem 1 is the particular instance $d=0$.

We prove the theorem by explicitly constructing $f\left(n ; F_{d}\right)$ and verifying its properties. For simplicity assume $F_{d}$ consists of all members $\left(x_{1}, x_{2}, \ldots\right.$, $x_{n}$ ) of $B_{n}$ such that $x_{i}=0$ for $d+1 \leq i \leq n$; any other $d$-dimensional face can be treated similarly. The cube $B_{n}$ can be written as a Cartesian product $B_{d} \times B_{n-d}$, i.e., a binary sequence of length $n$ is uniquely expressible as a sequence of length $d$ followed by one of length $n-d$. With this notation, we use the functions introduced before theorem 1 to define

$$
f\left(n ; F_{d}\right)=\bigcup\left\{B_{d} \times\{Z\}: Z \epsilon f(n-d)\right\}=B_{d} \times f(n-d) .
$$

Each set $B_{d} \times\{Z\}$ is easily shown to be a $d$ dimensional face of $B_{n}$ and is therefore connected; $F_{d}$ itself is obtained as $B_{d} \times\left\{0_{n-d}\right\}$ where $0_{n-d}$ is a sequence of $n-d$ zeros. Since $f(n-d)$ has $2^{n-d-1}$ members (see theorem 1 ), there are $2^{n-d-1}$ sets $B_{d} \times\{\boldsymbol{Z}\}$. To see that these sets are the components of $f\left(n ; F_{d}\right)$, it suffices to observe that any two members $Z, Z^{\prime}$ of the isolated function $f(n-d)$ differ in at least two positions, so that no member of $B_{d} \times\{\boldsymbol{Z}\}$ can be a neighbor of any member of $B_{d} \times\left\{Z^{\prime}\right\}$.

The complement of $f(n-d)$ in $B_{n-d}$ is the isolated set $g(n-d)$ which has $2^{n-d-1}$ members. Therefore the complement of $f\left(n ; F_{d}\right)$ in $B_{n}$ is

$$
g\left(n ; F_{d}\right)=\bigcup\left\{B_{d} \times\{Z\}: Z \epsilon g(n-d)\right\}=B_{d} \times g(n-d)
$$

whose components are the $2^{n-d-1} d$-dimensional faces $B_{d} \times\{\boldsymbol{Z}\}$ of $B_{n}$ with $Z \in g(n-d)$. Thus theorem 3 is proved.

Before proving the next theorem, it is convenient to collect several elementary facts as a lemma.

Lemma 3. Let $\mathrm{f}_{0}$ and $\mathrm{f}_{1}$ be the projections of $a$ cellular function $\mathrm{f}$ of $\mathrm{k}+1$ variables. Then

(a) if $\mathrm{h}$ is a component of $\mathrm{f}$, then either $\mathrm{h}_{0}=\phi$ or $\mathrm{h}_{1}=\phi$ or $\mathrm{h}_{0}=\mathrm{h}_{1}$,

(b) for $\mathrm{i}=0$ and $\mathrm{i}=1$, the components of $\mathrm{f}_{1}$ are

the nonvoid projections $\mathrm{h}_{1}$ of the components $\mathrm{h}$ of $\mathrm{f}$,

(c) $\mathrm{f}_{0}$ and $\mathrm{f}_{1}$ are cellular if nonempty, and

(d) if component $\mathrm{p}$ of $\mathrm{f}_{0}$ meets component $\mathrm{q}$ of $\mathrm{f}_{1}$,

then $\mathrm{p}=\mathrm{q}=\mathrm{h}_{0}=\mathrm{h}_{1}$ for some component $\mathrm{h}$ of $\mathrm{f}$.

To begin the proof, let $h$ be any component of $f$. Since $f$ is cellular, for some $d$ there exist $S_{h} \subset\{1,2, \ldots$, $k, k+1\}$ with $\mathrm{k}+1-d$ members, and a binary sequence $\left\{c_{i}: i \epsilon S_{h}\right\}$, such that $h$ consists of all members $\left(x_{1}, \ldots, x_{k+1}\right)$ of $B_{k+1}$ obeying

$$
x_{i}=c_{i} \text { for all } i \epsilon S_{h} \text {. }
$$

If $k+1 \epsilon S_{h}$ and $c_{k+1}=0$, then $h_{1}=\phi$ and $h_{0}$ consists of all members $\left(x_{1}, \ldots, x_{k}\right)$ of $B_{k}$ such that

$$
x_{i}=c_{i} \text { for all } i \epsilon S_{h}-\{k+1\} .
$$

If $k+1 \epsilon S_{h}$ and $c_{k+1}=1$, then $h_{0}=\phi$ and ${ }^{\top} h_{1}$ consists of all members $\left(x_{1}, \ldots, x_{k}\right)$ of $B_{k}$ such that

$$
x_{i}=c_{i} \text { for all } i \in S_{h}-\{k+1\} .
$$

If $k+1 \epsilon\{1, \ldots, k+1\}-S_{h}$, then $h$ is the disjoint union of the set of members of $B_{k+1}$ such that

$$
x_{k+1}=0, x_{i}=c_{i} \text { for all } i \epsilon S_{h}
$$

and the set of members of $B_{k+1}$ such that

$$
x_{k+1}=1, x_{i}=c_{i} \text { for all } i \in S_{h} ;
$$

thus $h_{0}=h_{1}$ consists of all members of $B_{k}$ such that

$$
x_{i}=c_{i} \text { for all } i \epsilon S_{h} \text {. }
$$

This proves (a) of the lemma, and also shows that $h_{0}$ and $h_{1}$ are faces of $B_{k}$ and therefore cells of $f_{0}$ and $f_{1}$ respectively. 
To prove (b) of the lemma for $i=0$ (the proof for $i=1$ is similar), it now suffices to show that if $h_{0}$ and $h_{0}^{*}$ are nonempty projections of distinct components $h$ and $h^{*}$ of $f$, then no member $X$ of $h_{0}$ is a neighbor of any member $X^{*}$ of $h_{0}^{*}$. If such a "neighboring" occurred, however, then the member $X \times\{0\}$ of $h$ would be a neighbor of the member $X^{*} \times\{0\}$ of $h^{*}$, which is impossible. Thus (b) holds; (c) follows from (b) and the fact (proved incidentally in the last paragraph) that the projections of a face are again faces.

Now suppose a component $p$ of $f_{0}$ and a component $q$ of $f_{1}$ have a member $X$ in common. By (b), there exist components $h_{p}$ and $h_{q}$ of $f$ such that $\left(h_{p}\right)_{0}=p$ and $\left(h_{q}\right)_{1}=q$. Thus $X \times\{0\}$ is in $h_{p}$ and its neighbor $X \times\{1\}$ is in $h_{q}$, so that $h_{p}=h_{q}=h$, say. It follows from (a) that $p=q$, so (d) is proved.

TheOREM 4. The maximum number of members in a cellular Boolean function of $\mathrm{n}$ variables (other than $\left.\mathrm{B}_{\mathrm{n}}\right)$ is $2^{\mathrm{n}-1}$. For $0 \leq \mathrm{d}<\mathrm{n}$ and each d-dimensional face $\mathrm{F}_{\mathrm{d}}$ of $\mathrm{B}_{\mathrm{n}}, \mathrm{f}\left(\mathrm{n} ; \mathrm{F}_{\mathrm{d}}\right)$ is the only cellular function with $2^{\mathrm{n}-1}$ members which has $\mathrm{F}_{\mathrm{a}}$ as a component.

This is clear for $n=1$; assume it true for $n=k$ and consider the case $n=k+1$. Let $V_{k+1}$ be the maximum number of members in a cellular function, other than $B_{k+1}$, of $k+1$ variables; $V_{k}$ is defined similarly. It follows from theorem 3 that $V_{k+1} \geq 2^{k}$. To prove the opposite inequality, let $f$ be a cellular function of $k+1$ variables with $V_{k+1}$ members. If either of $f_{0}$ or $f_{1}$ is $B_{k}$ then by (d) of lemma 3 the other must be empty, since otherwise we would have $f_{0}=f_{1}=B_{k}$ and thus $f=B_{k+1}$. Thus if $f_{0}=B_{k}$ then $f_{1}=\phi$ and $f=B_{k} \times\{0\}$, while if $f_{1}=B_{k}$ then $f_{0}=\phi$ and $f=B_{k} \times\{1\}$; in either case $V_{k+1}=|f|=2^{k}$. If both $f_{0}$ and $f_{1}$ are proper subsets of $B_{k}$ then by the inductive hypothesis and (c) of lemma 3 we have

$$
V_{k+1}=|f|=\left|f_{0}\right|+\left|f_{1}\right| \leq V_{k}+V_{k}=2^{k-1}+2^{k-1}=2^{k},
$$

completing the proof that $V_{n}=2^{n-1}$ for all $n$.

Now let $F_{d}$ be a $d$-dimensional face of $B_{k+1}$, where $0 \leq d<k+1$, and let $f$ be a cellular Boolean function of $k+1$ variables with $2^{k}$ members and $F_{d}$ as a component. For simplicity we again use the representation $B_{k+1}=B_{d} \times B_{k+1-d}$ and assume $F_{d}=B_{d} \times\left\{0_{k+1-d}\right\}$, where $0_{k+1-d}$ is a sequence of $k+1-d$ zeros. ${ }^{10 \mathrm{a}}$

If $f_{0}=B_{k}$ then (see the last paragraph)

$$
f=B_{k} \times\{0\}=f\left(k+1 ; F_{k}\right)=f\left(k+1 ; F_{d}\right)
$$

where the last equation holds since $F_{d}$ is to be a component and $B_{k} \times\{0\}$ has $F_{k}$ as sole component. If $f_{1}=B_{k}$ then (see the last paragraph) $f_{0}=\phi$, which is impossible since $f_{0}$ contains the nonempty set ${ }^{11}$ $\left(F_{d}\right)_{0}=B_{d} \times\left\{0_{k-d}\right\}$. So we can assume $f_{0}$ and $f_{1}$ are proper cellular functions of $k$ variables; by (3), $f_{0}$ and $f_{1}$ each have $2^{k-1}$ members, so that by inductive

\footnotetext{
10a For consistency it is important that this representation and assumption match the corresponding ones in the proof of theorem 3, so that the explicit formula for $f\left(n: F_{d}\right)$ introduced in that proof can validly be used below.

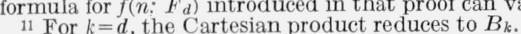

hypothesis and (b) and (c) of lemma 3,

$$
f_{0}=f\left(k ;\left(F_{d}\right)_{0}\right) .
$$

In the next paragraph we will show that $f_{1}=B_{k}-f_{0}$; it follows that

$$
f_{1}=g\left(k ;\left(F_{d}\right)_{0}\right)
$$

and thus by eq (2) that

$$
f=\left(f\left(k ;\left(F_{d}\right)_{0}\right) \times\{0\}\right) \cup\left(g\left(k ;\left(F_{d}\right)_{0}\right) \times\{1\}\right) .
$$

From the explicit formulas for $f\left(n ; F_{d}\right)$ and $g\left(n ; F_{d}\right)$ given in the proof of theorem 3 , we have

$$
\begin{aligned}
f\left(k ;\left(F_{d}\right)_{0}\right) \times\{0\} & =\left[\bigcup\left\{B_{d} \times\{Z\}: Z \epsilon f(k-d)\right\}\right] \times\{0\} \\
& =\bigcup\left\{B_{d} \times\{Z \times\{0\}\}: Z \epsilon f(k-d)\right\} \\
& =B_{d} \times(f(k-d) \times\{0\}),
\end{aligned}
$$

where we have used the fact $\left(F_{d}\right)_{0}=B_{d} \times\left\{0_{k-d}\right\}$, and similarly

$$
g\left(k ;\left(F_{d}\right)_{0}\right) \times\{1\}=B_{d} \times(g(k-d) \times\{1\}) .
$$

Thus

$$
\begin{aligned}
f & =B_{d} \times[(f(k-d) \times\{0\}) \cup(g(k-d) \times\{1\})] \\
& =B_{d} \times f(k+1-d)=f\left(k+1 ; F_{d}\right),
\end{aligned}
$$

as was to be proved.

It remains to prove that $f_{1}=B_{k}-f_{0}$. Since $f_{0}$ and $f_{1}$ each have $2^{k-1}$ members, it suffices to prove $f_{1} \subset B_{k}-f_{0}$, i.e., that no component $p$ of $f_{0}$ meets any component $q$ of $f_{1}$. If such a meeting occurred, then by (d) of lemma 3 we have $p=q=h_{0}=h_{1}$ for some component $h$ of $f$, and by (b) of lemma $3 p$ is a component of $f_{1}$ as well as $f_{0}$. By (c) of lemma 3, $f_{0}$ and $f_{1}$ are both cellular, and by induction hypothesis there is only one cellular function of $k$ variables with $2^{k-1}$ members having $p$ as a component. Therefore $f_{1}=f_{0}$, so that $\left(F_{d}\right)_{0}$ is a component of $f_{1}$ as well as of $f_{0}=f\left(k ;\left(F_{d}\right)_{0}\right)$. Thus

$$
\begin{aligned}
B_{d} \times\left\{0_{k-d}\right\} \times & B_{1} \\
& =\left[B_{d} \times\left\{0_{k-d}\right\} \times\{0\}\right] \cup\left[B_{d} \times\left\{0_{k-d}\right\} \times\{1\}\right] \\
& =\left[\left(F_{d}\right)_{0} \times\{0\}\right] \cup\left[\left(F_{d}\right)_{0} \times\{1\}\right] \\
& \subset\left[f_{0} \times\{0\}\right] \cup\left[f_{1} \times\{1\}\right]=f ;
\end{aligned}
$$

since the first set is a face of $B_{k+1}$ which properly contains $F_{d}=B_{d} \times\left\{0_{k+1-d}\right\}$, we have a contradiction to the hypothesis that $F_{d}$ is a component of $f$. This completes the proof of theorem 4 .

The last two theorems yield the following statement:

Corollary. A cellular Boolean function of $\mathrm{n}$ variables (other than $\mathrm{B}_{\mathrm{n}}$ ) with the maximum number of members has all its component cells of the same dimension, d, and its complement is again such a function (with the same "d"). There are $2\left(\begin{array}{l}\mathrm{n} \\ \mathrm{d}\end{array}\right)$ such functions 
for each $\mathrm{d}<\mathrm{n}$, and thus in all there are $2\left(2^{\mathrm{n}}-1\right)$ cellular functions of $\mathrm{n}$ variables with the maximum number of members.

The number $2\left(\begin{array}{l}n \\ d\end{array}\right)$ is obtained by dividing the number $2^{n-d}\left(\begin{array}{l}n \\ d\end{array}\right)$ of $d$-dimensional faces of $B_{n}$ by the number $2^{n-d-1}$ of these faces appearing in a maximum cellular function.

\section{Perfect Gray Codes}

An even simpler proof of lemma 2 and theorem 1, in section 2, can be given if we take as known the existence of a Gray code, i.e., an enumeration $\left\{X_{i}: 1 \leq i \leq 2^{n}\right\}$ of the members of $B_{n}$ such that $X_{i}$ and $X_{i+1}$ are neighbors for $1 \leq i \leq 2^{n}=N$, and $X_{N}$ and $X_{1}$ are neighbors. From this it is obvious that an isolated set cannot contain both $X_{i}$ and $X_{i+1}$, or both $X_{N}$ and $X_{1}$, and so has at most $2^{n-1}$ members. It is also clear that the sets

$$
F(n)=\left\{X_{i}: i \text { is even }\right\}, G(n)=\left\{X_{i}: i \text { is odd }\right\},
$$

if they are isolated, are the unique maximum isolated functions of $n$ variables. But the defining property of a Gray code shows that the parity of the number of 1 's in $X_{i}$ is opposite to that for $X_{i+1}$, so that the number of 1's in every member of $F(n)$ has the same parity, while the opposite parity holds for all members of $G(n)$. Thus either $F(n)=f(n)$ and $G(n)=g(n)$, or $F(n)=g(n)$ and $G(n)=g(n)$; since $f(n)$ and $g(n)$ are easily proved to be isolated the same is true of $F(n)$ and $G(n)$, so that the conclusions of lemma 2 and theorem 1 have been established.

The inductive technique used in section 2 to prove lemma 2 and theorem 1, and in section 3 to prove theorem 4, can also be employed to establish the existence of a Gray code on $\dot{B}_{n}$ for all $n \geq 1$. We shall actually prove a stronger result, namely the existence of a class of Gray codes (to be called perfect) with certain additional properties which have been found helpful in aiding visualization. For example ((a) of theorem 5), for each $d$ with $1 \leq d \leq n$ such a code partitions into $2^{n-d}$ successive subpaths, each essentially a perfect Gray code on some $d$-dimensional face of $B_{n}$. Also a rather explicit description can be given of the positions (in a perfect Gray code) of the $n$ neighbors of any member of $B_{n}$ whose position in the code is given; see corollary 1 to theorem 7 .

For $0 \leq d \leq n$ and $1 \leq j \leq 2^{n-d}=N(n-d)$, it is convenient to define

$$
S_{j}^{d}=\left\{i:(j-1) 2^{d}<i \leq j 2^{d}\right\},
$$

a subset of $\left\{1,2,3, \ldots, 2^{n}=N(n)\right\}$. For any subset $S$ of $S_{2 \nu-1}^{d}$, we call the subset

$$
S^{\prime}=\left\{i:(2 \nu-1) 2^{d+1}+1-i \epsilon S\right\}
$$

of $S_{2 \nu}^{d}$ the reflection of $S$ in $(2 \nu-1) 2^{d}$, while for any subset $S$ of $S_{2 \nu}^{d}$ the reflection $S^{\prime}$ of $S$ in $(2 \nu-1) 2^{d}$ is the subset of $S_{2 \nu-1}^{d}$ defined by the same formula. Here $0 \leq d<n$ and $1 \leq \nu \leq N(n-d-1)$. Familiarity with the reflected binary number system should provide partial motivation for this definition.
For any enumeration $\left\{X_{i}: 1 \leq i \leq 2^{n}=N(n)\right\}$ of the members of $B_{n}$, and any subset $\bar{T}$ of $\{1,2,3, \ldots$, $N(n)\}$, let

$$
X(T)=\left\{X_{i}: i \epsilon T\right\} .
$$

The enumeration will be called a perfect Gray code if, for each $d$ with $0 \leq d<n$, for each $\nu$ with $1 \leq \nu \leq$ $N(n-d-1)$, and for each subset $S$ of either $S_{2 \nu-1}^{d}$ or $S_{2 \nu}^{d}$ such that $X(S)$ is a $\delta$-dimensional face of $B_{n}$ for some $\delta \leq d$, the set $X\left(S^{\prime}\right)$ corresponding to the reflection $S^{\prime}$ of $S$ in $(2 \nu-1) 2^{d}$ is also a $\delta$-dimensional face, and furthermore $X(S) \cup X\left(S^{\prime}\right)$ is a $(\delta+1)$ dimensional face.

To indicate the implications of this fairly complicated definition, we collect some properties of perfect Gray codes in the next theorem, in which the notation

is employed.

$$
F_{j}^{d}=X\left(S_{j}^{d}\right)
$$

Theorem 5. If $\left\{\mathrm{X}_{1}: 1 \leq \mathrm{i} \leq \mathrm{N}(\mathrm{n})\right\}$ is a perfect Gray code on $\mathrm{B}_{\mathrm{n}}$, then

(a) $\mathrm{F}_{\mathrm{j}}^{\mathrm{d}}$ is a d-dimensional face,

(b) $\mathrm{F}_{\mathrm{j}}^{\mathrm{d}} \cup \mathrm{F}_{\mathrm{j}+1}^{\mathrm{d}}$ is a $(\mathrm{d}+1)$-dimensional face,

(c) $\mathrm{F}_{\mathrm{j}}^{\mathrm{d}} \cup \mathrm{F}_{\mathrm{N}(\mathrm{n}-\mathrm{d})+1-\mathrm{j}}^{\mathrm{d}}$ is a $(\mathrm{d}+1)$-dimensional face.

First (a) will be proved for $0 \leq d \leq n$. It is clearly true for $d=0$; assume it true for $d=k<n$, and consider the case $d=k+1$. Observe that

$$
S_{j}^{k+1}=S_{2 j-1}^{k} \cup S_{2 j}^{k}
$$

and that each disjunct is the other's reflection in $(2 j-1) 2^{d}$. Since by induction hypothesis $F_{2 j-1}^{k}$ is a $k$-dimensional face, it follows from the definition of "perfectness" that $F_{j}^{k+1}$ is a $(k+1)$-dimensional face. This completes the inductive proof of (a).

To prove (c), it now suffices to observe that $S_{N(n-d)+1-1}^{d}$ is the reflection of $S_{j}^{d}$ in $N(n-1)$. To prove (b), factor $j$ as

$$
j=2^{a} b<N(n-d) \quad(a \geq 0, b \text { odd })
$$

and observe that $S_{j}^{d}$ is a subset of $S_{b}^{a+d}$ whose reflection in $b 2^{a+d}$ is $S_{j+1}^{d}$.

By setting $d=0$ in (b) of theorem 5, and also in (c) with $j=1$, we see that a perfect Gray code is indeed a Gray code. An imperfect Gray code on $B_{3}$, where for example $F_{1}^{2}$ is not a face, is given by
$(0,0,0)$,
$(1,0,0)$,
$(1,0,1)$,
$(1,1,1)$
$(1,1,0)$,
$(0,1,0)$,
$(0,1,1)$,
$(0,0,1)$.

Next we prove the existence of a perfect Gray code on $B_{n}$; in analogy with theorem 3 it will in fact be shown that such a code can be chosen to "begin" with any preassigned face $F$ of $B_{n}$. Because the formal proof which follows is somewhat forbidding, we summarize the geometric idea first: $B_{n}$ is considered as made up of two copies of $B_{n-1}$ (for simplicity, $B_{n-1} \times\{0\}$ and $\left.B_{n-1} \times\{1\}\right)$, the first copy containing $F$; the perfect Gray code on $B_{n}$ is constructed by choosing an appropriate perfect Gray code on $B_{n-1}$ (which exists by induction hypothesis), tracing it out in $B_{n-1} \times\{0\}$ and then tracing it out in 
reverse order in $B_{n-1} \times\{1\}$. When $F=\phi$ this construction agrees with one given by Gilbert (op. cit. in footnote $9 \mathrm{~b}$ ), and if iterated for $n>1$ it gives the particular perfect Gray codes which Gilbert termed the "conventional" ones. ${ }^{12}$

Theorem 6. Let $\mathrm{F}$ be a $\mathrm{d}(0)$-dimensional face of $\mathrm{B}_{\mathrm{n}}$, with $0 \leq \mathrm{d}(0) \leq \mathrm{n}$. There exists a perfect Gray code on $\mathrm{B}_{\mathrm{n}}$ for which $\mathrm{F}_{1}^{\mathrm{d}}(0)=\mathrm{F}$.

This is clearly true for $n=1$; assume it true for $n=k$ and consider the case $n=k+1$. If $d(0)=k+1$, so that $F=B_{k+1}$, then we are simply to prove the existence of a perfect Gray code on $\bar{B}_{k+1}$, which will follow from the analysis for $d(0) \leq k$. So suppose $d(0) \leq k ;$ to simplify notation we can assume $F \subset \overline{B_{k}} \times\{0\}$, so that $F$ is given in terms of its projection $F_{0}$ as $F=F_{0} \times\{0\}$.

It is easy to prove that $F_{0}$ is a $d(0)$-dimensional face of $B_{k}$. By inductive hypothesis, there is a perfect Gray code $\left\{Y_{i}: 1 \leq i \leq N(k)\right\}$ on $B_{k}$ for which

$$
F_{0}=\left\{Y_{i}: 1 \leq i \leq 2^{d(0)}\right\} \text {. }
$$

Now define an enumeration $\left\{X_{i}: 1 \leq i \leq N(k+1)\right\}$ of $B_{k+1}$ by

$$
\begin{gathered}
X_{i}=Y_{i} \times\{0\} \text { if } 1 \leq i \leq N(k), \\
X_{i}=Y_{N(k+1)+1-i} \times\{1\} \text { if } N(k)<i \leq N(k+1) .
\end{gathered}
$$

We shall show that this enumeration is the desired perfect Gray code. The following notation will be used:

$$
\begin{aligned}
& X(S)=\left\{X_{i}: i \epsilon S\right\}, \\
& Y(R)=\left\{Y_{i}: i \epsilon R\right\} \text {, } \\
& S_{j}^{d}=\left\{i:(j-1) 2^{d}<i \leq j 2^{d}\right\} \quad(0 \leq d \leq k+1, \\
& 1 \leq j \leq N(k+1-d)) \text {, } \\
& R_{j}^{d}=\left\{i:(j-1) 2^{d}<i \leq j 2^{d}\right\}(0 \leq d \leq k, \\
& F_{j}^{d}=X\left(S_{j}^{d}\right), \\
& G_{j}^{d}=Y\left(R_{j}^{d}\right) . \\
& 1 \leq j \leq N(k-d))
\end{aligned}
$$

First, it is clear that

$$
F=F_{0} \times\{0\}=G_{1}^{a(0)} \times\{0\}=F_{1}^{d(0)},
$$

as desired. Second, consider any $d$ with $0 \leq d \leq k$, any $\nu$ with $1 \leq \nu \leq N(k-d)$, and any subset $S$ of either $S_{2 \nu-1}^{d}$ or $\bar{S}_{2 \nu}^{d}$ such that $X(S)$ is a $\delta$-dimensional face of $B_{k+1}$ for some $\delta \leq d$. Let $S^{\prime}$ be the reflection of $S$ in $(2 \nu-1) 2^{d}$. The proof will be completed by showing that $X\left(S^{\prime}\right)$ is a $\delta$-dimensional face, and $X(S) \cup X\left(S^{\prime}\right)$ a $(\delta+1)$-dimensional face. It will be assumed that $S$ is a subset of $S_{2 \nu-1}^{a}$ rather than $S_{2 \nu}^{u}$; the details for the other case are analogous.

Suppose first that $\nu \leq N(k-d-1)$. Then $X_{i}=$ $Y_{i} \times\{0\}$ for each $i \in S \cup S^{\prime}$. Therefore

$$
\begin{gathered}
X(S)=Y(S) \times\{0\}, \quad X\left(S^{\prime}\right)=Y\left(S^{\prime}\right) \times\{0\}, \\
X(S) \cup X\left(S^{\prime}\right)=\left(Y(S) \cup Y\left(S^{\prime}\right)\right) \times\{0\} .
\end{gathered}
$$

${ }^{12}$ In theorem 6 below we write $d(0)$ instead of $d_{0}$ for typographical convenience.
The first of these equations shows that $Y(S)$ is a $\delta$-dimensional face of $B_{k}$. so that $Y\left(S^{\prime}\right)$ is a $\delta$-dimensional face and $Y(S) \cup Y\left(S^{\prime}\right)$ a $(\delta+1)$-dimensional face; the second and third equations then yield the same results for $X(S)$ and $X(S) \cup X\left(S^{\prime}\right)$.

Next suppose $\nu>N(k-d-1)$ and $d<k$. Then

$$
X_{i}=Y_{N(k+1)+1-i} \times\{1\}
$$

for each $i \in S \cup S^{\prime}$. Let $R$ and $R^{\prime}$ be the respective reflections of $S$ and $S^{\prime}$ in $N(k)$, so that

$$
\begin{gathered}
X(S)=Y(R) \times\{1\}, \quad X\left(S^{\prime}\right)=Y\left(R^{\prime}\right) \times\{1\} \\
X(S) \cup X\left(S^{\prime}\right)=\left(Y(R) \cup Y\left(R^{\prime}\right)\right) \times\{1\} .
\end{gathered}
$$

The first of these equations shows that $Y(R)$ is a $\delta$-dimensional face of $B_{k}$. It is readily verified that $R^{\prime}$ is the reflection of $R$ in

$$
\left[2\left(2^{k-d}-(\nu-1)\right)-1\right] 2^{d},
$$

so that $Y\left(R^{\prime}\right)$ is a $\delta$-dimensional face and $Y(R) \cup Y\left(R^{\prime}\right)$ a $(\delta+1)$-dimensional face; the second and third equations then yield the same results for $X\left(S^{\prime}\right)$ and $X(S) \cup X\left(S^{\prime}\right)$.

The final situation is $d=k$, so that $\nu=1$. Here

$$
\begin{aligned}
& X_{i}=Y_{i} \times\{0\} \text { for } i \epsilon S, \\
& X_{i}=Y_{N(k+1)+1-i} \times\{1\} \text { for } i \epsilon S^{\prime}
\end{aligned}
$$

so that

$$
\begin{gathered}
X(S)=Y(S) \times\{0\} \quad X\left(S^{\prime}\right)=Y(S) \times\{1\}, \\
X(S) \cup X\left(S^{\prime}\right)=Y(S) \times\{0,1\},
\end{gathered}
$$

and the result follows.

The next theorem gives more detail on the structure of perfect Gray codes.

Theorem 7. Let $\left\{\mathrm{X}_{1}: 1 \leq \mathrm{i} \leq \mathrm{N}(\mathrm{n})\right\}$ be a perfect Gray code on $\mathrm{B}_{\mathrm{n}}$, and $\mathrm{T}$ a set of $2^{\delta+1}$ elements of $\{1,2, \ldots, \mathrm{N}(\mathrm{n})\}$. If $\mathrm{X}(\mathrm{T})$ is a $(\delta+1)$-dimensional face of $\mathrm{B}_{\mathrm{n}}$, then there exists $\mathrm{d}$ with $\delta \leq \mathrm{d}<\mathrm{n}, \nu$ with $1 \leq \nu \leq \mathrm{N}(\mathrm{n}-\mathrm{d}-1)$, and a partition $\overline{\mathrm{T}}=\mathrm{S} \cup \mathrm{S}^{\prime}$ such that $\mathrm{S}$ is a subset of either $\mathrm{S}_{2 v-1}^{d}$ or $\mathrm{S}_{2}^{d}, \mathrm{~S}^{\prime}$ is the reflection of $\mathrm{S}$ in $(2 \nu-1) 2^{\mathrm{d}}$, and $\mathrm{X}(\mathrm{S})$ is a $\delta$-dimensional face.

This is clear for $n=1$; assume it true for $n=k$, and consider the case $n=k+1$. By (a) of Theorem 5, $F_{1}^{k}$ is a $k$-dimensional face of $B_{k+1}$, and to simplify notation we may assume $F_{1}^{k}=B_{k} \times\{0\}$. Thus $F_{2}^{k}=B_{k} \times\{1\}$. If we define $Y_{i}$ to be the projection $\left(X_{i}\right)_{0}$ for $1 \leq i \leq N(k)$, then by (c) of theorem 5 $X_{N(k+1)+1-i} \epsilon B_{k} \times\{1\}$ is a neighbor of $X_{i} \epsilon B_{k} \times\{0\}$, which implies that $Y_{i}=\left(X_{N(k+1)+1-i}\right)_{1}$. That is,

$$
\begin{aligned}
& X_{i}=Y_{i} \times\{0\} \text { for } 1 \leq i \leq N(k) \\
& X_{i}=Y_{N(k+1)+1-i} \times\{1\} \text { for } N(k)<i \leq N(k+1) .
\end{aligned}
$$

That $\left\{Y_{i}: 1 \leq i \leq N(k)\right\}$ is a perfect Gray code on $B_{k}$ follows easily from the corresponding fact for $\left\{X_{i}: 1 \leq i \leq N(k+1)\right\}$ and the first of the last two equations.

Suppose first that $T \subset S_{1}^{k}$. Then

$$
X(T)=Y(T) \times\{0\}
$$


and so $Y(T)$ is a $(\delta+1)$-dimensional face of $B_{k}$. By induction hypothesis there exist $d$ with $\delta \leq d<\dot{k}$, $\nu$ with $1 \leq \nu \leq N(k-d-1)$, and a partition $T=S \cup S^{\prime}$ such that $S$ is a subset of either $S_{2 v-1}^{d}$ or $S_{22}^{d}, S^{\prime}$ is the reflection of $S$ in $(2 \nu-1) 2^{d}$, and $Y(S)$ is a $\delta$-dimensional face of $B_{k}$. Thus

$$
X(S)=Y(S) \times\{0\}
$$

is a $\delta$-dimensional face of $B_{k+1}$, as desired.

Next suppose $T \subset S_{2}^{k}$. Let $T^{\prime}$ be the reflection of $T$ in $N(k)$; then

$$
X(T)=Y\left(T^{\prime}\right) \times\{1\}
$$

and so $Y\left(T^{\prime}\right)$ is a $(\delta+1)$-dimensional face of $B_{k}$. By induction hypothesis there exist $d$ with $\delta \leq d<k$, $\nu$ with $1 \leq \nu \leq N(k-d-1)$, and a partition $T^{\prime}=S \cup S^{\prime}$ such that $S$ is a subset of either $S_{2 \nu-1}^{d}$ or $S_{2 \nu}^{d}, S^{\prime}$ is the reflection of $S$ in $(2 \nu-1) 2^{d}$, and $Y(S)$ is a $\delta$-dimensional face of $B_{k}$. Let $R$ and $R^{\prime}$ be the respective reflections of $S$ and $S^{\prime}$ in $N(k)$, so that we have a partition $T=R \cup R^{\prime}$, and

$$
X(R)=Y(S) \times\{1\}
$$

is a $\delta$-dimensional face of $B_{k+1}$. Since $R$ is a subset of either $S_{2 \mu-1}^{d}$ or $S_{2 \mu}^{d}$ where

$$
\mu=2^{k-d}-\nu+1,
$$

and $R^{\prime}$ is the reflection of $R$ in $(2 \mu-1) 2^{d}$, again matters are as desired.

Finally, suppose $T$ meets both $S_{1}^{k}$ and $S_{2}^{k}$. Then $X(T)$, as a $(\delta+1)$-dimensional face of $B_{k+1}$ which meets both $B_{k} \times\{0\}$ and $B_{k} \times\{1\}$, can easily be shown to have the form

$$
X(T)=F \times\{0,1\}
$$

where $F$ is a $\delta$-dimensional face of $B_{k}$. Say $F=Y(S)$ where $S \subset S_{1}^{k}$. It follows that

$$
T \cap S_{1}^{k}=S, \quad T \cap S_{2}^{k}=S^{\prime}
$$

where $S^{\prime}$ is the reflection of $S$ in $N(k)$. Since $X(S)=F \times\{0\}$ is a $\delta$-dimensional face of $B_{k+1}$, the proof is complete.

Corollary 1. Let $\left\{\mathrm{X}_{1}: 1 \leq \mathrm{i} \leq \mathrm{N}(\mathrm{n})\right\}$ be a perfect Gray code on $\mathrm{B}_{\mathrm{n}}$. Then the $\mathrm{n}$ neighbors of $\mathrm{X}_{1}$ are the $\left\{\mathrm{X}_{\mathrm{k}(\mathrm{d})}: 0 \leq \mathrm{d}<\mathrm{n}\right\}$ defined by

$$
\mathrm{k}(\mathrm{d})=(2 \nu(\mathrm{d})-1) 2^{\mathrm{d}+1}+1-\mathrm{i}
$$

where $\nu(\mathrm{d})$ is defined by

$$
(2 \nu(\mathrm{d})-2) 2^{\mathrm{d}}<\mathrm{i} \leq 2 \nu(\mathrm{d}) 2^{\mathrm{d}} .
$$

This follows from theorem 7 with $\delta=0$.

Corollary 2. Let $\left\{\mathrm{X}_{1}: 1 \leq \mathrm{i} \leq \mathrm{N}(\mathrm{n})\right\}$ be a perfect Gray code on $\mathrm{B}_{n}$. If $\mathrm{j}(1)$ and $\mathrm{j}(2)$ are distinct but have the same parity, then no member of $\mathrm{F}_{\mathrm{j}(1)}^{\delta}$ is a neighbor of a member of $\mathrm{F}_{\mathrm{j}(2)}^{\delta}$.
For, suppose a member $X_{i}$ of $F_{j(1)}^{\delta}$ and a member $X_{k}$ of $F_{j(2)}^{\delta}$ were neighbors, so that

$$
\begin{aligned}
& (j(1)-1) 2^{\delta}+1 \leq i \leq j(1) 2^{\delta}, \\
& (j(2)-1) 2^{\delta}+1 \leq k \leq j(2) 2^{\delta},
\end{aligned}
$$

and by corollary 1 there exist $d$ with $0 \leq d<n$, and $\nu=\nu(d)$, such that

and

$$
i+k=(2 \nu-1) 2^{d+1}+1
$$

$$
(\nu-1) 2^{d+1}+1 \leq i \leq \nu 2^{d+1},
$$

which imply that

$$
(\nu-1) 2^{d+1}+1 \leq k \leq \nu 2^{d+1} .
$$

On the one hand, we have

$$
\begin{aligned}
(j(2)-1) 2^{\delta}+1-j(1) 2^{\delta} & \leq k-i \\
& =(i+k)-2 i \\
& \leq\left[(2 \nu-1) 2^{d+1}+1\right] \\
& \quad-2\left[(\nu-1) 2^{d+1}+1\right]
\end{aligned}
$$

so that

$$
[j(2)-j(1)-1] 2^{\delta}+1 \leq 2^{d+1}-1
$$

and therefore

$$
j(2)-j(1)<2^{d+1-\delta}+1 .
$$

On the other hand by interchanging $i$ with $k$ and $j(1)$ with $j(2)$ in this argument, we obtain

$$
j(1)-j(2)<2^{d+1-\delta}+1 .
$$

Since the hypotheses on $j(1)$ and $j(2)$ imply $|j(2)-j(1)| \geq 2$, the last two displays imply that $d+1-\delta>0$. But then we would have

$$
\begin{aligned}
{\left[(j(1)-1) 2^{\delta}+1\right]+\left[(j(2)-1) 2^{\delta}+1\right] } & \leq i+k \\
& =(2 \nu-1) 2^{d+1}+1 \\
& =i+k \\
& \leq j(1) 2^{\delta}+j(2) 2^{\delta}
\end{aligned}
$$

so that

$$
\begin{gathered}
{[j(1)+j(2)-2] 2^{\delta}+2 \leq(2 \nu-1) 2^{d+1}+1 \leq[j(1)+j(2)] 2^{\delta},} \\
{[j(1)+j(2)-2] 2^{\delta}<(2 \nu-1) 2^{d+1}<[j(1)+j(2)] 2^{\delta},} \\
j(1)+j(2)-2<(2 \nu-1) 2^{d+1-\delta}<j(1)+j(2),
\end{gathered}
$$

with the hypotheses implying that the middle term (which lies between the consecutive even integers constituting the extreme terms) is an even integer. Since this is impossible, the corollary is proved.

Just as the existence of ordinary Gray codes provides an alternative approach to the material of section 2 (see the beginning of the present section), so the existence and properties of perfect Gray codes 
provide an alternative approach to the material of section 3 . Suppose for example that $F_{d}$ is a $d$-dimensional face of $B_{n}$, with $0 \leq d<n$. Then by theorem 6 there is a perfect Gray code on $B_{n}$ with $F_{d}=F_{1}^{d}$, and theorem 3 can be quickly proved by setting

$$
\begin{aligned}
& f\left(n ; F_{d}\right)=\bigcup\left\{F_{j}^{d}: 1 \leq j \leq N(n-d), j \text { is odd }\right\}, \\
& g\left(n ; F_{d}\right)=\bigcup\left\{F_{j}^{d}: 1 \leq j \leq N(n-d), j \text { is even }\right\}
\end{aligned}
$$

and invoking the last corollary (with $d$ as $\delta$ ) together with (a) of theorem 5 .

We conclude by showing that "essentially" there is only one perfect Gray code on $B_{n}$, which must therefore be the "conventional" one mentioned earlier. To make this precise, observe that as a "discrete cube," $B_{n}$ has as its natural group of symmetries the hyperoctahedral group $\mathscr{O}_{n}$ whose $(n !) 2^{n}$ elements can be represented as the ordered pairs $(\sigma, \pi)$ where $\sigma$ is a subset of $\{1,2,3, \ldots, n\}$ and $\pi$ is a permutation of $\{1,2,3, \ldots, n\}$. The operation of $(\sigma, \pi)$ on a member $X=\left(x_{1}, \ldots, x_{n}\right)$ of $B_{n}$ is the member $X^{\prime}$ defined by

$$
\begin{aligned}
x_{j}^{\prime} & =1-x_{\pi(j)} \text { if } j \epsilon \sigma, \\
x_{j}^{\prime} & =x_{\pi(j)} \text { if } j \epsilon\{1,2,3, \ldots, n\}-\sigma .
\end{aligned}
$$

If $\left\{X_{i}: 1 \leq i \leq N(n)\right\}$ and $\left\{X_{i}^{\prime}: 1 \leq i \leq N(n)\right\}$ are perfect Gray codes on $B_{n}$, we call them equivalent in case (with an obvious notation)

$$
(\sigma, \pi)\left(X_{i}\right)=X_{i}^{\prime} \text { for } 1 \leq i \leq N(n)
$$

for some $(\sigma, \pi)$ in $\mathscr{O}_{n}$.

Theorem 8. Any two perfect Gray codes on $\mathrm{B}_{\mathrm{n}}$ are equivalent.

This is clear for $n=1$; assume it true for $n=k$, and consider the case $n=k+1$. Let $\left\{X_{i}: 1 \leq i \leq N(k+1)\right\}$ and $\left\{X_{i}^{\prime}: 1 \leq i \leq N(k+1)\right\}$ be perfect Gray codes on $B_{k+1}$. By theorem $5 X\left(S_{1}^{k}\right)$ and $X^{\prime}\left(S_{1}^{k}\right)$ are $k$-dimensional faces of $B_{k+1}$, so that there exist elements $r$ and $t$ of $\{1,2, \ldots, k+1\}$, and numbers $c$ and $c^{\prime}$ each either zero or one, for which

$$
\begin{aligned}
& X\left(S_{1}^{k}\right)=\left\{X: X \epsilon B_{k+1}, x_{r}=c\right\}, \\
& X^{\prime}\left(S_{1}^{k}\right)=\left\{X: X \epsilon B_{k+1}, x_{t}=c^{\prime}\right\} .
\end{aligned}
$$

Therefore one-to-one correspondences between $B_{k}$, and each of $X\left(S_{1}^{k}\right)$ and $X^{\prime}\left(S_{1}^{k}\right)$, are set up by the functions

$$
g: X\left(S_{1}^{k}\right) \rightarrow B_{k}, \quad g^{\prime}: X^{\prime}\left(S_{1}^{k}\right) \rightarrow B_{k}
$$

defined by

$$
\begin{aligned}
& g(X)=\left(x_{\rho(1)}, x_{\rho(2)}, \ldots, x_{\rho(k)}\right), \\
& g^{\prime}(X)=\left(x_{\tau(1)}, x_{\tau(2)}, \ldots, x_{\tau(k)}\right)
\end{aligned}
$$

where $X=\left(x_{1}, \ldots, x_{k+1}\right)$ and the permutations $\rho$ and $\tau$ of $\{1,2, \ldots, k+1\}$ are given by $\rho(j)=j$ if $j<r, \quad \rho(j)=j+1$ if $r \leq j \leq k, \quad \rho(k+1)=r$, $\tau(j)=j$ if $j<t, \quad \tau(j)=j+1$ if $t \leq j \leq k, \quad \tau(k+1)=t$.

It is trivial to verify that $g$ transforms each $d$-dimensional face of $B_{k+1}$ which lies wholly in $X\left(S_{1}^{k}\right)$ into a $d$-dimensional face of $B_{k}$, while $g^{\prime}$ does the same for those faces of $B_{k+1}$ which lie wholly in $X^{\prime}\left(S_{1}^{k}\right)$. Therefore

$$
\left\{g\left(X_{i}\right): 1 \leq i \leq N(k)\right\} \text { and }\left\{g^{\prime}\left(X_{i}^{\prime}\right): 1 \leq i \leq N(k)\right\}
$$

are perfect Gray codes on $B_{k}$, so by induction hypothesis there exist a subset $\sigma$ of $\{1,2, \ldots, k\}$ and a permutation $\pi$ of $\{1,2, \ldots, k\}$ such that

$$
(\sigma, \pi)\left(g\left(X_{i}\right)\right)=g^{\prime}\left(X_{i}^{\prime}\right) \text { for } 1 \leq i \leq N(k) .
$$

If $x_{i j}$ and $x_{i j}^{\prime}$ denote the $j$ th entries of $X_{i}$ and $X_{i}^{\prime}$ respectively, then this can be written as

$$
\begin{aligned}
& x_{i, \tau(j)}^{\prime}=1-x_{i, \rho \pi(j)} \text { if } j \epsilon \sigma \\
& x_{i, \tau(j)}^{\prime}=x_{i, \rho \pi(j)} \quad \text { if } j \epsilon\{1,2, \ldots, k\}-\sigma
\end{aligned}
$$

for $1 \leq j \leq k$ and $1 \leq i \leq N(k)$.

Now form the permutation $\pi^{\prime}$ of $\{1,2, \ldots, k+1\}$ defined by $\pi^{\prime}(t)=r$ and

$$
\pi^{\prime}(j)=\rho \pi \tau^{-1}(j) \text { for } j \neq t,
$$

as well as the subset $\sigma^{\prime}$ of $\{1,2, \ldots, k+1\}$ defined by

$$
\begin{aligned}
& \sigma^{\prime}=\tau(\sigma) \cup\{t\} \text { if } c \neq c^{\prime}, \\
& \sigma^{\prime}=\tau(\sigma) \text { if } c=c^{\prime} .
\end{aligned}
$$

Then it follows easily that

$$
\left(\sigma^{\prime}, \pi^{\prime}\right)\left(X_{i}\right)=X_{i}^{\prime} \text { for } 1 \leq i \leq N(k),
$$

and this remains true for $N(k)<i \leq N(k+1)$ because

$$
\begin{aligned}
& x_{N(k+1)+1-i, j}=x_{i j} \text { if } j \neq r, \\
& x_{N(k+1)+1-i, r}=1-x_{i r}, \\
& x^{\prime}{ }_{N(k+1)+1-i, j}=x_{i j}^{\prime} \text { if } j \neq t, \\
& x^{\prime}{ }_{N(k+1)+1-i, t}=1-x_{i t}^{\prime}
\end{aligned}
$$

for $1 \leq i \leq N(k)$. Thus the two given perfect Gray codes on $B_{k+1}$ are equivalent.

It is a pleasure to acknowledge helpful suggestions by K. Goldberg and E. Johnson (NBS Numerical Analysis Section) and R. Kirsch (NBS Components and Techniques Section). 